\title{
Photonics Active Filters Based on SiC Multilayer Structures: A Two Stage Active Circuit
}

\author{
Manuel A. Vieira ${ }^{1,2}$, Alessandro Fantoni ${ }^{1,2}$, Paula Louro ${ }^{1,2}$, \\ Adolfo S. Garção ${ }^{2,3}$, and Manuela Vieira ${ }^{1,2,3}$ \\ ${ }^{1}$ Electronics Telecommunications and Computer Dept, ISEL, Lisbon, Portugal \\ ${ }^{2}$ CTS-UNINOVA, Quinta da Torre, 2829-516, Caparica, Portugal \\ ${ }^{3}$ DEE-FCT-UNL, Quinta da Torre, 2829-516, Caparica, Portugal
}

\begin{abstract}
Characteristics of tunable wavelength filters based on a-SiC:H multilayered stacked cells are studied both theoretically and experimentally. Results show that the light-activated photonic device combines the demultiplexing operation with the simultaneous photodetection and self amplification of an optical signal. The sensor is a bias wavelength current-controlled device that make use of changes in the wavelength of the background to control the power delivered to the load, acting a photonic active filter. Its gain depends on the background wavelength that controls the electrical field profile across the device.
\end{abstract}

Keywords: Amorphous $\mathrm{Si} / \mathrm{SiC}$ photodiodes, photonic, optoelectronic, demultiplexer devices, optical filters.

\section{Introduction}

There has been much research on semiconductor devices as elements for optical communication when a band or frequency needs to be filtered from a wider range of mixed signals [1]. Active filter circuits can be designed. Amorphous silicon carbon tandem structures, through an adequate engineering design of the multiple layers' thickness, absorption coefficient and dark conductivities can accomplish this function [1]. Here the manipulation of the magnitude is achieved by changing the wavelength (color channels) of the modulated lights and its frequency under appropriated wavelength backgrounds [2] and can be described in terms of a wavelength controlled photo-capacitance.

Combined tunable converters based on $\mathrm{SiC}$ multilayer photonics active filters are analyzed and optimized for a fine tuning of a specific wavelength. The operation combines the properties of active high-pass and low-pass filter sections into a capacitive active band-pass filter. A capacitive active band-pass filter model supports the experimental data. A numerical optoelectronic simulation gives insight into the physics of the device.

\section{Contribution to Value Creation}

Systems that transmit, receive, and/or process intelligence require some form of modulation, which is the deliberate distortion of a carrier to impress intelligence (data) 
upon it, in a manner that allows the subsequent recovery of the information. By providing a complex illumination of the device combining radiations with different and complementary penetration depths it is possible to control the spectral response of the device.

A combined RGB wavelength router/switch optical network can increase the connectivity of an optical system in visible range using automatically reconfigurable circuit-switching solutions and reduce the number of signal demultiplexers and wavelength division switches required by conventional multi-stage switching node architecture It provides a low-cost solution to many aspects of optical and optoelectronic interconnection technologies and architectures according to their suitability of adoption in optical cross connects (OXCs), switches and routers. So, the conception of new devices based on new materials for optically switchable on $\mathrm{SiC}$ multilayer photonic structures in the visible spectrum is a demand in the direction of an increased potential for value creation.

\section{Experimental Details}

The active device is a glass/ITO/a-SiC:H (p-i-n)/ a-SiC:H(-p) /Si:H(-i)/SiC:H ($\mathrm{n}) / \mathrm{ITO}$ double heterostructure produced by PECVD. Deposition conditions are described elsewhere [3].

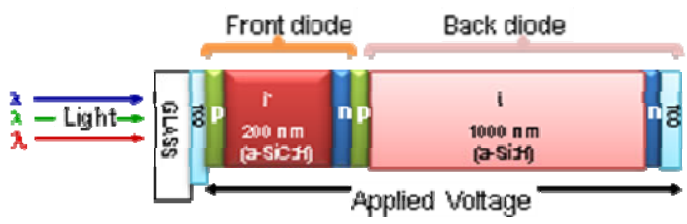

Fig. 1. Device configuration

The thickness $(200 \mathrm{~nm})$ and the absorption coefficient of the front photodiode (based on a-SiC:H) are optimized for short wavelength collection and long wavelength transmittance, and the thickness of the back one $(1000 \mathrm{~nm})$ adjusted to achieve full absorption in the intermediate wavelength region and high collection in the long wavelength spectral section. As a result, both front and back diodes act as optical filters confining, respectively, the short and the long wavelength optical carriers, while the intermediate ones are absorbed across both. In Fig. 1 the device configuration is depicted.

The device operates within the visible range using as input color channels (data) the wave square modulated light (external regulation of frequency and intensity) supplied by a red (R: $626 \mathrm{~nm} ; 51 \mu \mathrm{W} / \mathrm{cm}^{2}$ ), a green (G: $524 \mathrm{~nm} ; 73 \mu \mathrm{W} / \mathrm{cm}^{2}$ ) and a blue (B: $470 \mathrm{~nm} ; 115 \mu \mathrm{W} / \mathrm{cm}^{2}$ ) LED. Additionally, steady state red, green and blue illumination (background) was superimposed brought in LEDS driven at a constant current value (R: $290 \mu \mathrm{W} / \mathrm{cm}^{2}$, G: $150 \mu \mathrm{W} / \mathrm{cm}^{2}, \mathrm{~B}: 390 \mu \mathrm{W} / \mathrm{cm}^{2}$ ). Light was always impinging from the glass side. 


\section{Transfer Function Characteristics}

The transfer function magnitude (or gain) at each wavelength allows us to determine how well the optical filter can distinguish between signals at different wavelengths. In Fig. 2, the spectral photocurrent at $250 \mathrm{~Hz}$, is displayed under red, green and blue background irradiations (color symbols) and without it (black symbols). For comparison the normalized spectral photocurrent for the front, p-i'-n, and the back, p-i-n, photodiodes (lines) are superimposed. In Fig. 3 the gain, defined as the ratio between the spectral photocurrents, under red $\left(\alpha^{\mathrm{R}}\right)$, green $\left(\alpha^{\mathrm{G}}\right)$ and blue $\left(\alpha^{\mathrm{B}}\right)$ steady state illumination and without it (no background) are plotted at $250 \mathrm{~Hz}$ (lines) and $3500 \mathrm{~Hz}$ (symbols).

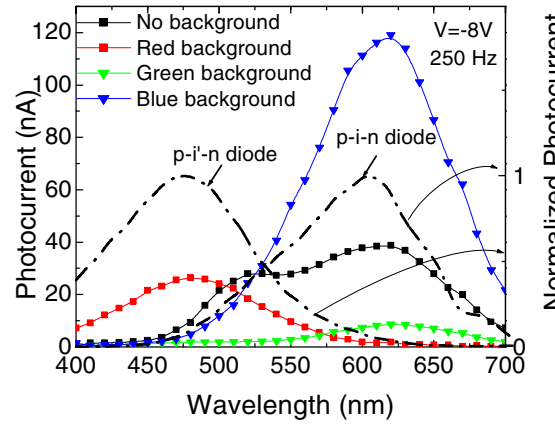

Fig. 2. Spectral photocurrent without and under different backgrounds (symbols). The normalized current of the front, $\mathrm{p}-\mathrm{i}$ '-n, and the back $\mathrm{p}-\mathrm{i}-\mathrm{n}$ photodiodes (lines) is superimposed

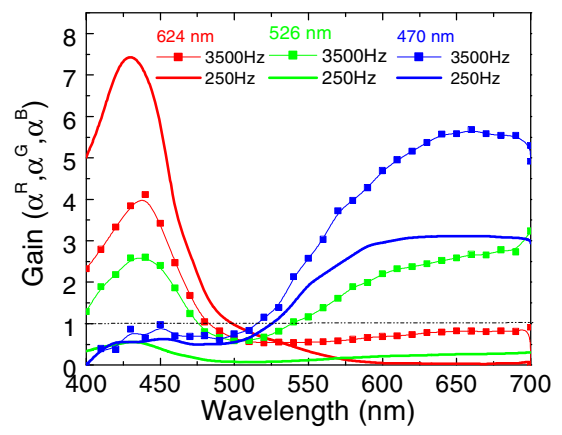

Fig. 3. Spectral gain under red $\left(\alpha^{\mathrm{R}}\right)$, green $\left(\alpha^{\mathrm{G}}\right)$, and blue $\left(\alpha^{\mathrm{B}}\right)$, optical bias for different frequencies

Data shows that each diode, separately, presents the typical response of single $\mathrm{p}-\mathrm{i}-\mathrm{n}$ cells with intrinsic layers based on a-SiC:H or a-Si:H materials, respectively. The front diode, based on a-SiC:H heterostructure, cuts the wavelengths higher than 550 $\mathrm{nm}$ while the back one, based on a-Si:H, rejects the ones lower than $500 \mathrm{~nm}$. The overall device presents a high spectral sensitivity in-between. Under red irradiation, the photocurrent is strongly enhanced at short wavelengths and disappears for wavelengths higher than $550 \mathrm{~nm}$, acting as a short-pass filter. Under blue irradiation the devices behaves as a long-pass filter for wavelengths higher than $550 \mathrm{~nm}$, blocking the shorter wavelengths. Under green irradiation the spectral response depends on the frequency. At $250 \mathrm{~Hz}$ the spectral sensitivity is strongly reduced while at $3500 \mathrm{~Hz}$ the device behaves as a band-stop active filter that screens out the medium wavelength range (green) enhancing only the photocurrent for wavelengths outside of that range. So, by changing the wavelength of the background the front and back diode sensitivities can be tuned.

Fig. 4 shows the spectral gain as a function of the frequency under red $\left(\alpha^{\mathrm{R}}\right)$, green $\left(\alpha^{\mathrm{G}}\right)$ and blue $\left(\alpha^{\mathrm{B}}\right)$ backgrounds at $624 \mathrm{~nm}\left(\alpha_{\mathrm{R}}\right.$, red channel), at $526 \mathrm{~nm}\left(\alpha_{\mathrm{G}}\right.$, green channel) and at $470 \mathrm{~nm}\left(\alpha_{\mathrm{B}}\right.$, blue channel). 
(a)

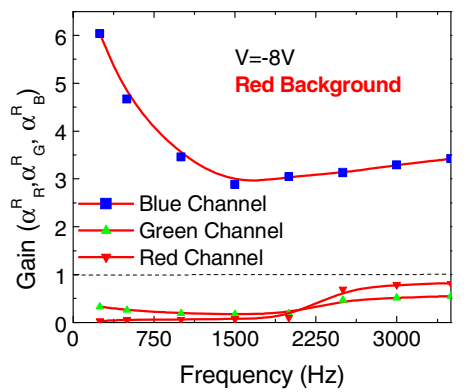

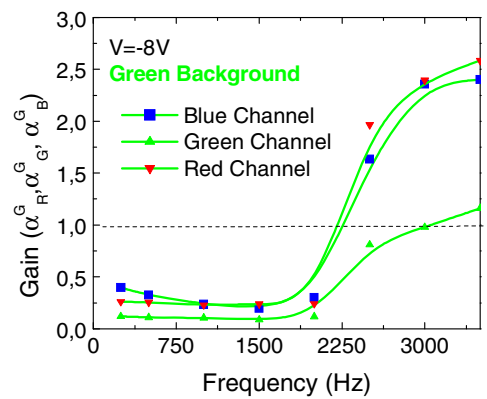

(b)

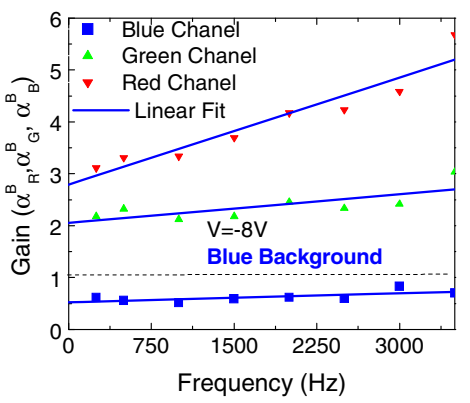

(c)

Fig. 4. Spectral gain as a function of the frequency at $624 \mathrm{~nm}$ (red channel), at $526 \mathrm{~nm}$ (green channel) and at $470 \mathrm{~nm}$ (blue channel) under red $(\alpha \mathrm{R})$, green $(\alpha \mathrm{G})$ and blue $(\alpha \mathrm{B})$ backgrounds. a) short-pass filter, b) band-stop filter, c) long-pass filter.

Under red and green irradiations two frequency regimes can be considered. One, for frequencies lower than $2000 \mathrm{~Hz}$, were either under red and green backgrounds the green and the red channel gains are very low $(<<1)$ and the blue gain is strongly enhanced $(>>1)$ under red background or reduced $(<1)$ under green irradiations. The other regime, for frequencies higher than $2000 \mathrm{~Hz}$, the gain increases with the frequency, gradually under red and quickly under green steady state illumination. Under blue, the gain increases slowly with the frequency being higher than one for the red and green channels and lower for the blue one. Consequently, under red irradiation (Fig. 4a) the transfer function has extra gain at short wavelengths (blue channel), than at longer wavelengths acting as a short-pass filter whatever the frequency. Under green background (Fig. 4b), the manipulation of amplitude is achieved by changing the frequency of the modulated lights. At high frequencies the device is a band-stop active filter that works to screen out wavelengths that are within a certain range (green channel), giving easy passage only all wavelengths below (blue channel) and above (red channel). In the low frequency regime all the amplitudes are quenched. Under blue steady state optical bias (Fig. 4c) the device behaves as a long-pass active filter that transmits and enhances the long wavelength photons (red and green channels) while blocking the shorter wavelengths (blue channel). 


\section{Numerical Simulation}

In order to understand the light filtering properties of the device, under different electrical and optical bias conditions, a simulation program ASCA-2D [4] was used having as input parameters the experimental data.

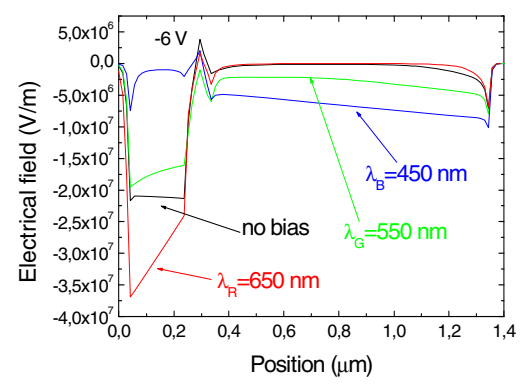

(a)

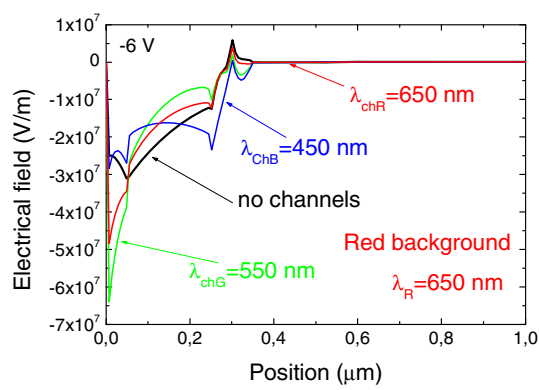

(b)

Fig. 5. Electric field profiles within the p-i'-n/p-i-n tandem structure. a) under different wavelengths backgrounds $\left(\lambda_{\mathrm{R}, \mathrm{G}, \mathrm{B}}\right)$ and without it, b) red background $\left(\lambda_{\mathrm{R}}\right)$ and different color channels $\left(\lambda_{\text {chR }}, \lambda_{\text {chG }}, \lambda_{\text {chB }}\right)$

Under negative applied voltage, in Fig. 5a, it is reported the electric field profile under different wavelengths backgrounds of the optical bias. In Fig. 5b, under red background, the three red, green and blue channels were added and the electrical field profile displayed.

Results show that the balance between the electrical field adjustments due to the non uniform absorption throughout the structure depends on the generation/recombination profiles at each background wavelength. The shallow penetration of the blue photons into the front diode, the deep penetration of the red photons exclusively into the back diode or the decay of the green absorption across both controls the sensor behavior. The external background interferes mainly with the less absorbing cell (the front under red, the back under blue and with both under green irradiations) [5]. Both the front and the back diodes are optically and electrically in series. Under steady state irradiation, to sustain the current across the device, the current at the less absorbing diode has to be adjusted through an increase of the electrical field and thus it becomes reverse biased (Fig. 5a). The superposition of a color channel will affect locally this field. Under red background (Fig. 5b), the blue channel increases the field intensity in the front diode and even reverse it at the internal interface increasing carrier collection. The red and the green channels change the field in an opposite way.

Frequency dependence of the device responsivity can be explained in terms of different induced background photo capacitances due to the transient charge accumulation (free and trapped) created in the top diode region. The photocapacitance can be assumed to be a measure of the density of photogenerated carriers in the space charge region. Fig. 6 shows the internal space charge profile within the complete device under different light 
wavelength. Charge accumulation is mainly localized at the internal interface between the two diodes. Under blue and red background light the charge accumulation is maximized for the complementary wavelength while under green background there is no marked difference between the space charge accumulation caused by the three signals and the background itself. This charge accumulation corresponds to a photo capacitance behavior of the internal recombination junction. Depending on the imposed background the different channel wavelengths produce a different displacement of the space charge distribution. This effect produces a background dependence of the device frequency response, allowing frequency filtering, or even the definition of an oscillatory frequency resonance. Combined with the series and parallel resistance of the diodes will result in the explicit definition of cut off frequencies for a frequency capacitive filters activated by the light background (Fig. 4).

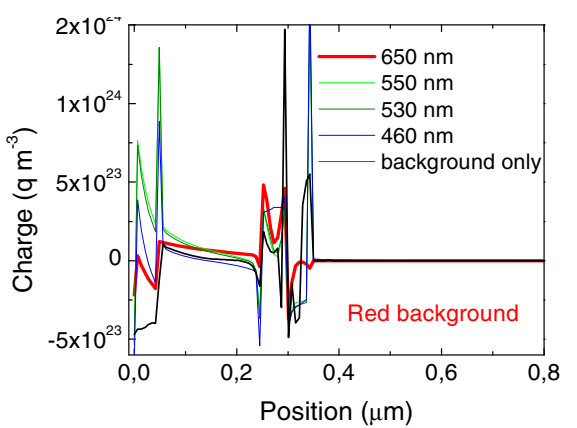

(a)

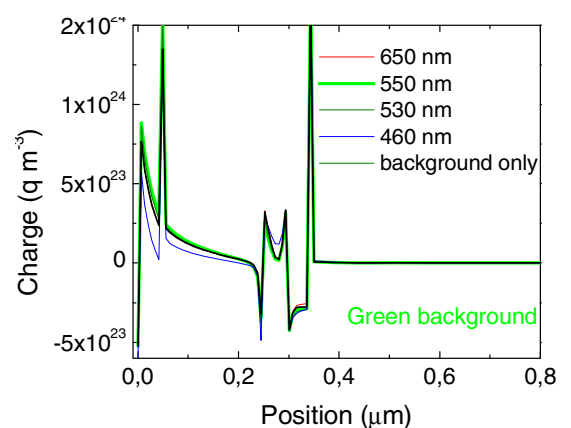

(b)

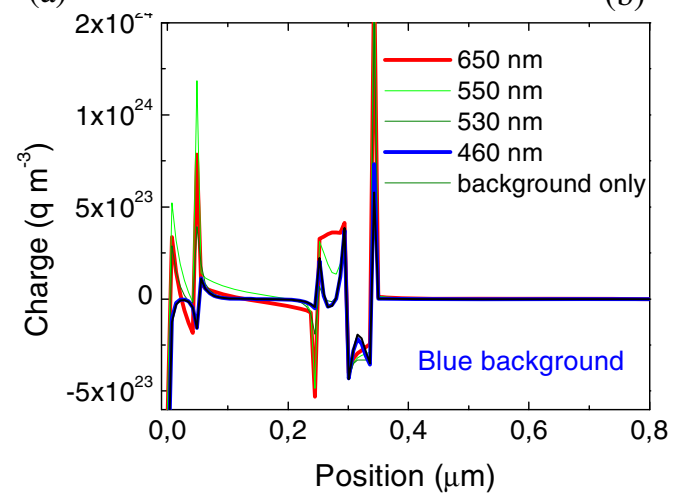

(c)

Fig. 6. Internal space charge profile within the device under different light wavelength. Results are shown under reverse bias and for different background illumination

\section{A Two Stage Active Circuit}

An optoelectronic model supported by the complete dynamical large signal EbersMoll model was developed [3] and is displayed in Fig. 7. The electrical model can be divided into two parallel sections (Fig. 7a): a short-pass filter (front phototransitor, 
$\mathrm{Q}_{1}$ ) and a long-pass filter (back phototransistor, $\mathrm{Q}_{2}$ ) sections. (Fig. 7b). The charge stored in the space-charge layers is modeled by the capacitor $\mathrm{C}_{1}$ and $\mathrm{C}_{2} . \mathrm{R}_{1}$ and $\mathrm{R}_{2}$ model the dynamical resistances of the internal and back junctions under different $\mathrm{dc}$ bias conditions (Fig. 5c).

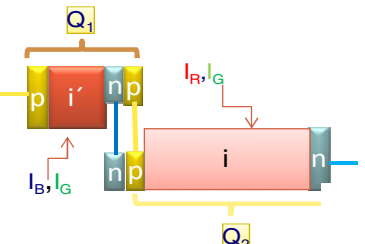

(a)
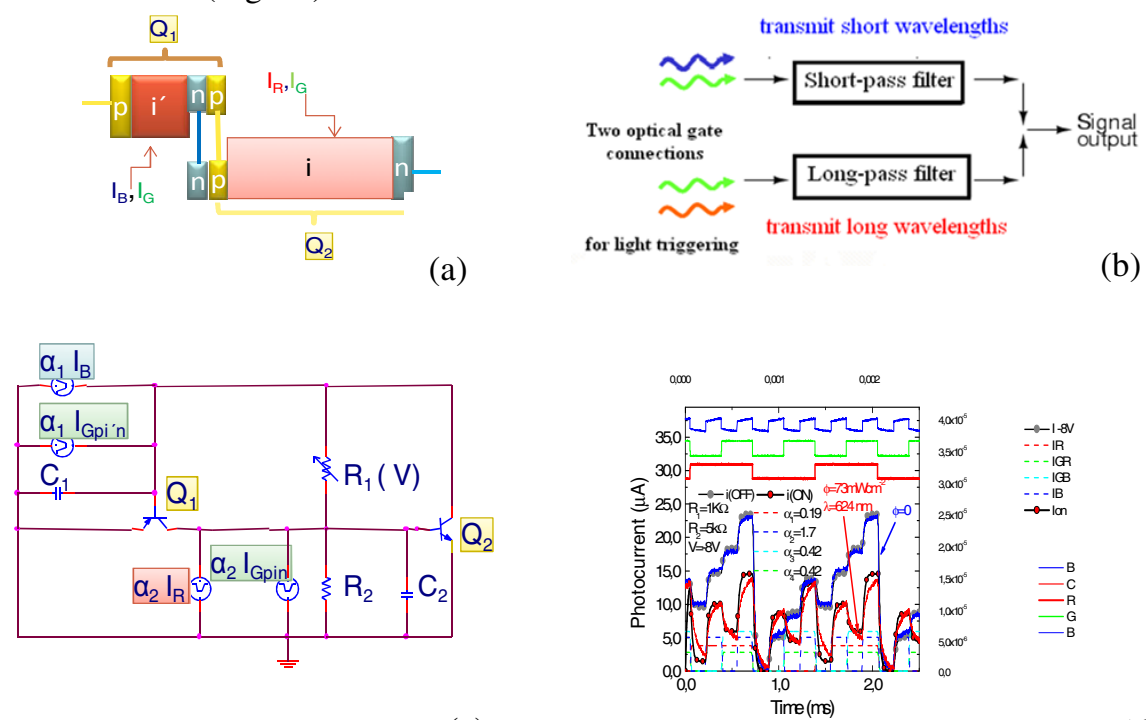

(c)

Fig. 7. a) Two connected transistor model, b) Two active capacitive filter sections and c) ac equivalent circuit. d) Output simulated waveform (symbols), current sources (dash lines) and experimental signals (solid lines), under negative $\left(\mathrm{R}_{1}=1 \mathrm{k} \Omega ;-8 \mathrm{~V}\right)$ without and with red background.

The operation is based upon the following principle: the flow of current through the resistor connecting the two transistor bases, $R_{1}$, is proportional to the difference in the voltages across both capacitors (charge storage buckets). To allow independent blue, red and green channels transmission and to take into account the wavelength background effect four square-wave current sources with different intensities are used; two of them, $\alpha_{1} I_{B}$ and $\alpha_{2} I_{R}$, with different frequencies to simulate the input blue and red channels and the other two, $\alpha_{1} \mathrm{I}_{\text {Gpi'n }}$ and $\alpha_{2} \mathrm{I}_{\text {Gpin }}$, with the same frequency but different intensities, to simulate the green channel due to its asymmetrical absorption across both front and back phototransistors. When the device is negatively biased, the base-emitter junction of both transistors are inversely polarized and conceived as phototransistors, taking, so, advantage of the amplifier action of adjacent collector junctions which are polarized directly. This results in a current gain proportional to the ratio between both collector currents. The amplifying elements, $\alpha_{1}$ and $\alpha_{2}$, control the spatial variation of the photocurrent and so of the photo capacitance of both front and back phototransistors and can provide gain if needed or attenuate unwanted wavelengths $(<1)$ while amplifying $(>1)$ desired ones. The values and the strategic placement of the resistors determine the basic shape of the output signals. 
To validate the model, in Fig. 7d, experimental (solid lines) and the simulated (symbols) waveform under negative bias and red background is shown. The bit sequences to drive the channels are shown in the top of the Fig.. The current sources are also displayed (dash lines). To simulate the red background, current sources intensities were multiplied by the on/off ratio between the input channels with and without optical bias (Fig. 3). A good agreement between experimental and simulated data was achieved. Under red background the expected optical amplification in the low wavelength range is observed due to the effect of the active multiple-feedback filter when the back diode is light triggered. The device behaves like an optoelectronic controlled transmission system that stores, amplifies and transports the minority carriers generated by the current pulses, through the capacitors $C_{1}$ and $C_{2}$. Depending on its wavelength, the optical bias changes the photo capacitance and so the voltages across one or both capacitors. The amplitude of the $a c$ current sources is affected by an $\alpha$ factor that takes into account the enhancement or quenching of the channels due to the steady state irradiation.

\section{Conclusions}

Light-activated photonic devices that combine the demultiplexing operation with the simultaneous photodetection and self amplification of an optical signal were presented. Depending on the wavelength of the external background they act either as a short- or a long- pass band filter or as a band-stop filter. A two stage active circuit and a numerical simulation support device operation. Results show the device is a currentcontrol device. It uses the photo capacitances to control the power delivered to the load acting as a state variable filter circuit. It combines the properties of active highpass and low-pass filter sections into a capacitive active filter.

\section{References}

1. Louro, P., Vieira, M., Vygranenko, Y., Fantoni, A., Fernandes, M., Lavareda, G., Carvalho, N.: Optical readout in pinpi'n and pini'p imagers: a comparison. In: Mat. Res. Soc. Symp. Proc., vol. 989, A12.04 (2007)

2. Vieira, M.A., Louro, P., Vieira, M., Fantoni, A., Garção, A.S.: Light-activated amplification in Si-C tandem devices: A capacitive active filter model. IEEE Sensors Journal (2011)

3. Vieira, M., Fantoni, A., Fernandes, M., Louro, P., Lavareda, G., Carvalho, C.N.: Bias sensitive multispectral structures for imaging applications. Thin Solid Films 515(19), 75667570 (2007)

4. Fantoni, A., Vieira, M., Martins, R.: Simulation of hydrogenated amorphous and microcrystalline silicon optoelectronic devices. Mathematics and Computers in Simulation 49, 381-401 (1999)

5. Vieira, M., Fantoni, A., Louro, P., Fernandes, M., Schwarz, R., Lavareda, G., Carvalho, C.N.: Vacuum 82(12), 1512-1516 (2008) 